Acta Cryst. (2002). A58 (Supplement), C271

\section{STUDIES ON THE GLIAL CELL-LINE DERIVED NEUROTROPHIC FACTOR (GDNF) CO-RECEPTOR a1}

V.-M. Leppanen ${ }^{1}$ M. Bespalov ${ }^{1}$ M. Saarma ${ }^{1}$ A. Goldman ${ }^{1}$

${ }^{1}$ University of Helsinki Institute of Biotechnology Viikinkaari 1 HELSINKI 00710 FINLAND

Glial cell line-derived neurotrophic factor (GDNF) is known to promote the survival and differentiation of many neuron populations. The receptor complex for GDNF was discovered to consist of the transmembrane RET receptor tyrosine kinase and a ligand-binding component, the GDNF co-receptor $\alpha 1$ (1). Binding studies $(1,2)$ suggest that GDNF serves mainly to dimerize the coreceptor, GFR $\alpha 1$, and interacts weakly, if at all, with RET in ternary complex. The GDNF signaling is beginning to be understood, but many questions remain open. Therefore, structural studies on the GFR $\alpha 1$ were initiated to address these questions at the molecular level. The crystal structure of GDNF has already been solved (3). GFR $\alpha 1$ has a signal peptide for secretion at the amino terminus, a glycosylphosphatidylinositol (GPI) -anchor signal at the Cterminus and three potential $\mathrm{N}$-glycosylation sites (1). Conserved cysteine patterning (4) suggests a putative domain structure with three homologous cysteine-rich domains, each about 100 residues long, bound together with hinge regions.

Baculovirus infected insect cells are being successfully used to produce rat GFR $\alpha 1$ for structural studies. Optimized suspension cultures in 100 to $200 \mathrm{ml}$ volumes repeatedly give mg-levels per liter of the 6His-tagged construct. A purification protocol, comprising of Ni-resin and gel filtration, has already been established. Vapor-diffusion techniques are being applied to obtain crystals suitable for structural studies.

References

1. Jing, S. et al. (1996). Cell 85:1113-

2. Scott, R., and Ibanez, C. (2001). J. Biol. Chem. 276:1450-

3. Eigenbrot, C., and Gerber, N. (1997). Nat. Struct. Biol. 4:435-

4. Lindahl, M. et al (2000). Mol. Cell. Neurosci. 15:522-

Keywords: GDNF, CO-RECEPTOR

\section{Acta Cryst. (2002). A58 (Supplement), C271 \\ CRYSTAL STRUCTURE OF THE PROTEASE DOMAIN OF A HEAT SHOCK PROTEASE}

K. K. Kim D. Y. Kim

Department of Molecular Cell Biology, SBRI Sungkyunkwan University School of Medicine SUWON 440-746 SOUTH KOREA

HtrA (high temperature requirement A), a periplasmic heat shock protein, is known to have molecular chaperone function at low temperatures and proteolytic activity at elevated temperatures. To investigate the mechanism of functional switch to protease, we have determined the crystal structure of the $\mathrm{N}$-terminal protease domain (PD) of HtrA from Thermotoga maritima. HtrA PD shares the same fold with chymotrypsin-like serine proteases but it contains an additional helical lid which blocks access of substrates to the active site. Furthermore, a catalytic triad and an oxyanion hole, which are necessary for protease activity, are not formed properly. On the surface of the lid, HtrA PD has hydrophobic patches that could be potential substrate binding sites for molecular chaperone activity. The crystal structure suggests that HtrA PD is not an active protease at current state. Therefore the activation of the proteolytic function of HtrA PD at elevated temperatures might occur by the conformational change which includes the opening of the helical lid to expose the active site and subsequent rearrangement of the catalytic triad and the oxyanion hole.

Keywords: CRYSTAL, PROTEASE, CHAPERONE
Acta Cryst. (2002). A58 (Supplement), C271

THE 1.9 A STRUCTURE OF $\alpha$-N-ACETYLGALACTOSAMINIDASE: MOLECULAR BASIS OF GLYCOSIDASE DEFICIENCY DISEASES S.C. Garman $^{1}$ L. Hannick ${ }^{1}$ A. Zhu ${ }^{2}$ D.N. Garboczi ${ }^{1}$

${ }^{1}$ Laboratory of Immunogenetics, National Institutes of Health National Institute of Allergy and Infectious Diseases Twinbrook 2, Room 10912441 Parklawn Drive ROCKVILLE MD 20852 USA ${ }^{2}$ Lindsley F. Kimball Research Institute, New York Blood Center

In the lysosome, glycosidases degrade glycolipids, glycoproteins, and oligosaccharides. Mutations in glycosidases cause disorders characterized by the deposition of undegraded carbohydrates. Schindler and Fabry diseases are caused by the incomplete degradation of carbohydrates with terminal $\alpha-\mathrm{N}$ acetylgalactosamine and $\alpha$-galactose, respectively. Here we present the x-ray structure of $\alpha-\mathrm{N}$-acetylgalactosaminidase ( $\alpha$-NAGAL, E.C. 3.2.1.49), the glycosidase that removes $\alpha-\mathrm{N}$-acetylgalactosamine, and the structure with bound ligand. The enzyme is a homodimer with each monomer comprised of two domains, a $(\beta / \alpha) 8$ barrel domain containing the active site and a domain with eight antiparallel $\beta$ strands. The active site features many aspartic acids, including one (D140) in position to act as a nuclephile and one (D201) in position to act as a general acid/base in the double displacement reaction mechanism. The active site residues of $\alpha$-NAGAL are conserved in the closely related enzyme $\alpha$-galactosidase A ( $\alpha$-GAL, E.C. 3.2.1.22), except for near where the substrates differ. The structure demonstrates the catalytic mechanisms of both enzymes and reveals the structural basis of mutations causing Schindler and Fabry diseases. As $\alpha$-NAGAL and $\alpha$-GAL produce type $\mathrm{O}$ universal donor blood from type $\mathrm{A}$ and type $\mathrm{B}$ blood, the $\alpha$-NAGAL structure will aid the engineering of improved enzymes for blood conversion.

Keywords: GLYCOSIDASE, SCHINDLER DISEASE, FABRY DISEASE

Acta Cryst. (2002). A58 (Supplement), C271

X-RAY STRUCTURAL ANALYSIS OF ALGINATE LYASE A1-III MUTANTS/SUBSTRATE COMPLEXES: ACTIVATION OF A CATALYTIC TYROSINE RESIDUE BY A FLEXIBLE LID LOOP $\underline{\text { B. Mikami }}{ }^{1}$ S. Suzuki ${ }^{1}$ H.-J. Yoon ${ }^{2}$ O. Miyake ${ }^{1}$ W. Hashimoto ${ }^{1}$ K. Murata ${ }^{1}$ ${ }^{1}$ Graduate School of Agriculture, Kyoto University Laboratory of Quality Design and Exploitation Gokasho UJI KYOTO 611-0011 JAPAN ${ }^{2}$ Division of Chemistry \& Molecular Engineering, Seoul National University

Alginate lyase A1-III from a Sphingomonas species A1 is a $\beta$-D-mannuronosyl linkage specific lyase and efficiently liquefies acetylated alginates produced by bacteria. The three-dimensional structure of A1-III was abundant in helices and had a deep tunnel-like cleft in a $(\alpha 6 / \alpha 5)$-barrel structure (1). It has been proposed that Y246 is a catalytic residue responsible for abstraction and donation of a hydrogen during catalysis (2). The structures of the mutant enzymes (H192A and Y246F) in the presence or absence of a tetrasaccharide substrate (4-deoxy-L-erythro-hex-4-enepyranosyluronate-mannuronatemannuronate-mannuronic acid) were determined by X-ray crystallography. In the crystal of H192A apo form, the lid loop (residues 64-85) takes a wide-open conformation, while it takes a closed conformation contacting with the substrate and the catalytic residues in the holo form. The tetrasaccharide was bound in the active cleft at subsites $-3+1$ from the non-reducing end by forming several hydrogen bonds and van der Waals interactions with protein atoms. Both Y246 and H192 were located near subsites -1 and +1 . Y246 OH atom is proximity both with an oxygen of glycosidic bond and a hydrogen of $\mathrm{C} 5$ of the sugar residue at +1 site. A strong hydrogen bond was found between $\mathrm{OH}$ of Y246 and $\mathrm{OH}$ of Y68, which suggests the stabilisation of ionised Y246 during catalysis.

References

(1) H.-J. Yoon et al., J. Mol Biol. 290, 505-514, 1999.

(2). H.-J. Yoon et al., J. Mol. Biol., 307, 9-16, 2001.

Keywords: ALGINATE LYASE ENZYME STRUCTURE CATALYTIC MECHANISM 